\title{
«Idea of a Perfect Commonwealth » ou le réalisme utopique de David Hume
}

\section{Gilles Robel}

\section{(2) OpenEdition}

\section{Journals}

Édition électronique

URL : http://journals.openedition.org/etudesecossaises/176

DOI : 10.4000/etudesecossaises. 176

ISSN : 1969-6337

\section{Éditeur}

UGA Éditions/Université Grenoble Alpes

\section{Édition imprimée}

Date de publication : 30 janvier 2008

Pagination : 9-29

ISBN : 978-2-84310-110-6

ISSN : $1240-1439$

\section{Référence électronique}

Gilles Robel, « «Idea of a Perfect Commonwealth » ou le réalisme utopique de David Hume », Études écossaises [En ligne], 11 | 2008, mis en ligne le 30 janvier 2009, consulté le 08 septembre 2020. URL : http://journals.openedition.org/etudesecossaises/176; DOI : https://doi.org/10.4000/ etudesecossaises.176

Ce document a été généré automatiquement le 8 septembre 2020.

(c) Études écossaises 


\title{
«Idea of a Perfect Commonwealth » ou le réalisme utopique de David Hume
}

\author{
Gilles Robel
}

1 David Hume n'est pas un auteur que l'on a coutume d'associer à l'idée d'utopie. En tant qu'empiriste, que sceptique et que représentant des Lumières «conservatrices » écossaises, l'auteur du Traité de la nature humaine serait plus volontiers qualifié de penseur réaliste, très méfiant à l'égard de toute proposition radicale de changement :

A established government has an inifinite advantage, by that very circumstance of its being established [...]. To tamper, therefore, in this affair, of try experiments merely upon the credit of supposed argument and philosophy, can never be the part of a wise magistrate, who will bear a reverence to what carries the marks of the age. (Essays, p. 512-513)

2 Aussi peut-il sembler étrange de constater qu'il publia dans le volume des Political Discourses paru en 1752 un projet utopique intitulé «Idea of a Perfect Commonwealth » d'où est tiré le passage qui précède. Second paradoxe, ce texte, qui s'inscrit dans une tradition de pensée républicaine et rend explicitement hommage à l'un de ses principaux théoriciens, James Harrington, remet en question les maximes essentielles du républicanisme du même Harrington. Au point qu'un commentateur de Hume, relevant ces « incohérences », écrivit qu'il ne fallait pas prendre Hume au sérieux et qualifia son utopie de "satire». (Conniff 1976, p. 101 et 1980, p. 382) Quel est donc le statut exact de cet essai? Pour le déterminer, nous examinerons d'abord brièvement le genre de l'utopie politique aux XVII et au XVIII ${ }^{e}$ siècle, en insistant sur Oceana de Harrington. Puis nous étudierons la typologie humienne des régimes politiques pour déterminer la place que Hume accorde au régime républicain avant d'examiner le contenu de son projet de commonwealth idéal et ce qui le rattache ou au contraire l'éloigne de la tradition républicaine. Nous 
montrerons enfin que la particularité de cette utopie est d'être une utopie réalisable, conforme en cela à la philosophie politique de Hume.

\section{L'utopie politique au XVIII siècle : la richesse ou la vertu}

3 Le genre de l'utopie est florissant au $\mathrm{xVIII}^{\mathrm{e}}$ siècle. Sa popularité s'explique par le développement des récits de voyage ou les progrès de la science, mais aussi par l'accroissement de la pauvreté ou enfin, à la fin du siècle, par la volonté de transformer radicalement les institutions, surtout en France (Claeys 1994, p. vii-ix). Les auteurs d'utopies présentent au lecteur des sociétés où règnent la vertu, la paix et l'égalité, mais ils adoptent deux approches bien distinctes selon qu'ils pensent que l'amélioration de la société est possible ou non. Ceux qui doutent de la possibilité d'améliorer les choses ont souvent recours à la satire. Gulliver's Travels (1726) de Jonathan Swift est l'exemple le plus célèbre d'une satire utopique qui dénonce les travers de la société tout en proposant, mais de façon ambiguë, un programme de réforme. Dans d'autres utopies non satiriques telles que Robinson Crusoe de Daniel Defoe (1717), c'est par un retour à une sorte d'État de Nature que l'idéal d'une société bien ordonnée est atteint par un individu qui finit par prendre en main sa destinée. De même The Island of Content (1709) présente une terre où le travail est inutile, où le vin est bon mais ne provoque pas l'ivresse et où règnent le bonheur et la piété. (Claeys 1994, p. 3-25) Il s'agit là d'utopies régressives. En revanche, certaines utopies présentent des modèles de gouvernement et des propositions concrètes de réforme. Ces utopies projectives ont en commun de dénoncer la corruption, question politique centrale au XVIII siècle (Langford 1992, p. 716). Elles stigmatisent le règne de l'argent dans les sociétés commerçantes, les inégalités, les trafics d'influence et enfin l'accroissement de la dette publique. Nombre de ces utopies sont le fait de membres du Country party ou de l'opposition tory à l'oligarchie whig․ Et nombre de ces utopies projectives s'inspirent des théories républicaines développées au XVII ${ }^{\mathrm{e}}$ siècle.

4 Le républicanisme classique ou l'humanisme civique sont un «langage » ou une idéologie héritée de l'antiquité grecque et romaine (Aristote, Polybe), réinterprétée par Machiavel à la Renaissance, et qui joue un rôle essentiel en Angleterre pendant la Guerre civile et en Écosse au siècle suivant :

Civic humanism denotes a style of thought... in which it is contended that the development of the individual towards self-fulfilment is possible only when the individual acts as a citizen, that is as a conscious and autonomous participant in an autonomous decision-taking political community, the polis or republic. (Pocock 1973, p. 82)

5 La tradition républicaine valorise plus que toute autre chose l'autonomie morale que les citoyens peuvent acquérir en manifestant leur virtù, c'est-à-dire en participant pleinement à la vie politique de la communauté, définie avant tout en termes constitutionnels ou institutionnels, et en défendant les libertés inscrites dans la Constitution, ce qui implique aussi la participation à la défense militaire de la communauté. Cette recherche de la vertu civique est souvent jugée incompatible avec l'essor du commerce, et le débat entre richesse et vertu forme un cadre interprétatif majeur de l'histoire intellectuelle des XVII ${ }^{e}$ 
et XVIII ${ }^{e}$ siècles : de façon très schématique, on trouve d'un côté le libéralisme, la modernité, la richesse, le commerce, la sociabilité, les armées de métier, l'interdépendance; de l'autre le républicanisme, les modèles républicains antiques, l'austérité, le rejet du luxe et de la corruption, le port des armes, la terre, l'indépendance ${ }^{2}$. De nombreuses utopies montrent ainsi que le goût du luxe et le développement du commerce sont une source de dépravation ${ }^{3}$. Cette vision de l'histoire, et cette opposition entre commerce et vertu républicaine théorisée notamment par Pocock, ont fait l'objet de critiques récentes : Steven Pincus montre ainsi que les républicains classiques développèrent dès les années 1640 une conception de l'intérêt commun qui était compatible avec la société commerciale. Toutefois ce n'est pas le cas dans l'une des utopies républicaines les plus célèbres, dont Hume s'inspire directement, à savoir Oceana de James Harrington ${ }^{4}$.

6 The Commonwealth of Océana parut en 1656 durant le Commonwealth, c'est-àdire le régime républicain instauré par Cromwell entre l'exécution de Charles I ${ }^{\text {er }}$ (1649) et la restauration de Charles II (1660). Harrington ne cherche pas tant à justifier la chute de la monarchie qu'à en expliquer les causes, et il présente une théorie et un modèle de gouvernement républicain qui se situe dans une Angleterre fictive mais aisément identifiable ${ }^{5}$. Ce régime idéal est une "république égalitaire » dans laquelle la citoyenneté dépend de la capacité des hommes à assurer leur indépendance par le port des armes et par la possession de terres suffisantes pour subvenir à leurs besoins. La terre, qui est selon Harrington la principale source de richesse, ne doit donc être monopolisée par personne et la Constitution vise à éviter les formes extrêmes d'inégalité qui engendrent des luttes partisanes entre riches et pauvres. L'égalité économique est assurée par la loi agraire, qui empêche que la propriété foncière soit concentrée entre quelques mains. Elle oblige les grands propriétaires à diviser équitablement leurs terres entre tous leurs fils, afin d'éviter tout retour au féodalisme. L'égalité politique est assurée par un système de rotation dans les trois Ordres d'élus que compte le gouvernement: équivalent politique de la circulation du sang ou du mouvement des astres, le roulement permet au corps entier des citoyens de se transformer en permanence en gouvernement, en évitant toute monopolisation du pouvoir. Le Sénat est composé de membres élus en raison de leur excellence (ils forment une "aristocratie de talents »), qui ont l'initiative des lois et en débattent; les citoyens sont représentés dans une Assemblée populaire qui examine et vote les lois; enfin les magistrats veillent à l'application des lois. Le Sénat et l'Assemblée populaire sont renouvelés par tiers tous les ans. Les magistrats sont élus pour un ou trois ans, et leur mandat ne peut être renouvelé qu'après une période d'interruption égale à la durée de leur mandat. Il existe une stricte séparation des pouvoirs. Ces principes doivent garantir la liberté et l'indépendance des citoyens de la république. 


\section{Le républicanisme de Hume}

7 Les utopies projectives au XVIII ${ }^{\mathrm{e}}$ siècle ne s'inscrivent pas toutes dans la tradition républicaine; en tant que whig sceptique, Hume critique l'oligarchie whig sans approuver l'opposition tory; enfin dans le débat entre la vertu et le commerce, entre l'antiquité et la modernité, Hume est indéniablement un «moderne»: il témoigne d'une plus grande confiance que beaucoup de penseurs éclairés dans la civilisation commerçante moderne ${ }^{6}$. C'est pourquoi il semble paradoxal que son modèle de constitution idéale soit de type républicain, et qu'il s'inspire directement du Oceana de Harrington. À quoi tient le choix du régime républicain? Et en quoi le commonwealth idéal de Hume se distingue-t-il de celui de Harrington et du républicanisme classique?

\section{Typologie des régimes politiques}

8 Bien qu'il paraisse considérer, dans son analyse de la justice et de l'allégeance, que se valent les gouvernements qui remplissent leurs fonctions de base, à savoir l'application des règles de la justice et le maintien de la stabilité de la société, Hume est loin de partager l'irénisme qu'exprime Pope dans les célèbres vers :

For forms of government let fools contest,

Whate'er is best admnister'd is best ${ }^{7}$.

9 Hume fait de la réfutation d'une telle attitude l'objet même de sa théorie politique : il est clair à ses yeux que tous les régimes ne se valent pas, ni dans l'absolu, ni de manière relative. Ainsi qu'il l'écrit dans l'essai «That Politics May Be Reduced To A Science » (1741) :

It is a question with several, whether there be any essential difference between one form of government and another? and, whether every form may not become good or bad, according as it is well or ill administered? Were it once admitted, that all governments are alike... most political disputes would be at an end, and all Zeal for one Constitution above another, must be esteemed mere bigotry and folly. But, though a friend to moderation, I cannot forbear condemning this sentiment... (Essays, p. 14-15).

10 Hume conteste particulièrement l'idée selon laquelle les institutions étant de valeur égale, ce seraient les humeurs des hommes politiques qui importent : il récuse une telle analyse à propos de Robert Walpole, accusé par les tories d'avoir corrompu la constitution.

11 La comparaison des différentes formes de régime politique est, pour Hume, la première tâche du savant politique qui cherche à éviter les généralisations hâtives. Sans se livrer à une classification systématique des types de gouvernement, Hume opère entre eux d'importantes distinctions. Il condamne les régimes arbitraires (barbarousmonarchies ou tyrannies) car ils ne remplissent que partiellement leur fonction de garant de la sécurité et de la liberté de leurs sujets, et freinent le développement des arts et des sciences ${ }^{8}$. Hume semble d'ailleurs considérer dans l'Histoire d'Angleterre que la République de Cromwell, après que le Lord Protecteur eut confié des pouvoirs étendus à douze majorsgénéraux entre août 1655 et janvier 1657 (c'est-à-dire précisément à l'époque où Harrington composa Oceana) ressemblait beaucoup à une tyrannie orientale. 
12 La définition du second type de gouvernement, les " monarchies civilisées » (civilized monarchies, appelées parfois simplement absolute monarchies), marque un trait original de la pensée de Hume et montre sa volonté de remettre en question les doctrines dominantes à l'époque, en particulier celle qu'il dénomme "whiggisme vulgaire ${ }^{10}$ ». Selon cette doctrine d'inspiration lockienne, qui dépasse le cadre du parti whig puisqu'on la retrouve chez un tory comme Bolingbroke, les monarchies absolues ne peuvent constituer une forme de gouvernement civil, car leurs sujets n'ont pas eu la possibilité de formuler de consentement à l'autorité du monarque ${ }^{11}$.

13 Contre cette longue tradition de pensée qui défend le contrat social, associe la république à la liberté, et la monarchie absolue à la tyrannie, Hume s'emploie à montrer qu'il n'y a pas d'incompatibilité entre ces monarchies et la défense des libertés civiques. Loin d'opposer les monarchies civilisées aux républiques, Hume pense que le développement des premières est lié à l'existence des secondes. Par un phénomène d'imitation et de contagion, les monarques absolus ont été contraints d'adopter certains principes républicains, si bien que tout en détenant un pouvoir absolu, ils ne gouvernent pas de manière arbitraire ; ils délèguent souvent leurs fonctions à des subordonnés qui agissent sous le contrôle de la loi ${ }^{12}$. L'avantage de ce type de régime est que le pouvoir y est très stable en raison de la transmission héréditaire de la Couronne et de l'absence de limites constitutionnelles étroites. Cela signifie que les groupes d'intérêt et les factions ne sont pas incités à rivaliser pour accroître leur influence, et que le roi n'a pas de raison constitutionnelle de favoriser l'un ou l'autre de ces groupes. Et puisque son autorité est respectée, le roi n'a pas non plus de raison d'exercer son pouvoir de manière particulièrement autoritaire. C'est bien sûr à la monarchie française que Hume songe lorsqu'il évoque ce type de régime, et il montre que ses sujets ne sont pas des "esclaves ${ }^{13}$ ». Pourtant, explique Hume, l'inconvénient majeur de ce type de régime est que tout dépend des qualités personnelles du monarque, c'est-à-dire que beaucoup est laissé au hasard ; or, l'histoire prouve que les monarques ne sont pas toujours irréprochables ${ }^{14}$. Il le juge donc inférieur aux « régimes libres ».

14 Les gouvernements populaires sont les «régimes libres» ou free governments, dont Hume fournit une définition dans l'essai «Of the Origin of Governement » :

A free government] admits a partition of power among several members, whose united authority is [...] commonly greater than that of any monarch ; but who [...] must act by general and equal law, that are previously known to all members, and to all their subjects (Essays, p. 40).

15 Deux régimes fonctionnent selon ce mode : les « républiques pures » (pure republics) comme la démocratie athénienne ou la Hollande, et les "monarchies mixtes » ou "monarchies limitées" (limited ou mixed monarchies) dont la Grande-Bretagne offre le seul et remarquable exemple moderne. Ces régimes se distinguent des monarchies civilisées par le fait que le pouvoir est séparé entre plusieurs corps, et que les principaux intérêts socio-économiques sont représentés dans l'une au moins de ces branches - en général le corps législatif. Cette séparation du pouvoir nécessite l'existence de règles précises, et le peuple est gouverné en fonction de lois générales et uniformes, sans être soumis aux 
décisions d'un seul ; si les juges et les dirigeants conservent un certain pouvoir discrétionnaire, celui-ci est cantonné dans d'étroites limites.

16 Le problème central de ces régimes mixtes est de maintenir un équilibre entre les différents corps qui les composent, ou plus précisément, de déterminer jusqu'à quel point les citoyens peuvent jouir de la liberté civique sans menacer l'autorité politique. Dans une monarchie mixte, il faut parvenir à limiter le pouvoir du roi sans pour autant le réduire à une impuissance complète : c'est un problème que Hume examine de manière approfondie à propos de la constitution britannique. Dans les républiques, il faut éviter que l'élément aristocratique ne soit dominé par l'élément populaire, et l'équilibre y est souvent précaire.

17 Si étrange que cela puisse paraître eu égard à son rejet du "whiggisme vulgaire ", la république constitue bien pour Hume la forme idéale de gouvernement. En effet, l'Etat de droit (« rule of law ») et la liberté ne peuvent se développer dans une monarchie barbare, alors qu'une république conduit nécessairement à la définition de lois générales, car elles sont les conditions de sa survie, comme Hume l'écrit dans "Of the Rise and Progress of Arts and Sciences» :

Though a republic should be barbarous, it necessarily, by an infallible operation, gives rise to LAW, even before mankind have made any considerable advances in the other sciences. From law arises security : From security curiosity: And from curiosity knowledge. The latter steps of this progress may be more accidental ; but the former are altogether necessary. A republic without laws can never have any duration (Essays, p. 118).

18 La république, même lorsque n'y règne pas le « rule of law » au sens strict du terme, est donc un facteur essentiel de civilisation, et c'est à ce titre qu'elle a la préférence de Hume. Le grand avantage des régimes libres, à ses yeux, est d'être plus favorables qu'une monarchie, fut-elle civilisée, au développement du commerce et de l'esprit d'entreprise. La raison principale en est le type de structure sociale et les valeurs que la monarchie encourage.

19 Dans le cas des régimes établis en Europe, Hume se réjouit de l'introduction, par imitation, d'éléments républicains dans la monarchie absolue française, mais il s'inquiète de leur poids excessif dans le régime britannique. S'il considère la république comme un régime idéal, Hume émet un certain nombre de réserves lorsqu'il examine le fonctionnement des régimes républicains historiques. Dans le cas des républiques antiques, il constate qu'il n'existait pas de moyen terme entre un pouvoir aristocratique autoritaire et un pouvoir démocratique anarchique et il s'emploie, dans l'essai « Of the Populousness of Ancient Nations ", à relativiser l'admiration de nombreux penseurs éclairés pour les républiques antiques au détriment des régimes modernes (Essays, p. 416). Hume est hostile aux républiques de forme démocratique car il estime que les régimes dans lesquels le peuple gouverne, que ce soit de manière directe ou indirecte, sont des régimes instables, propices à tous les enthousiasmes, et qui encouragent des comportements démagogiques chez leurs dirigeants. Le régime républicain, qu'il soit antique ou moderne, dégénère donc facilement en tyrannie: la manière dont il décrit les partisans de Cromwell, leur bellicisme et leur caractère factieux fait clairement écho à son 
évocation de la république athénienne ${ }^{15}$. Pour éviter que les républiques et les gouvernements libres ne sombrent dans le désordre, il faut y établir des constitutions solides et un certain nombre de garde-fous que Hume mentionne dans plusieurs essais et qui sont naturellement présents dans son commonwealth idéal. C'est ici qu'intervient le savant politique, conseiller du législateur, bien loin de l'irénisme de Pope (Essays, p. 24).

20 S'il ressort de sa typologie des régimes politiques que Hume a une légère préférence pour les régimes libres, et s'il rejoint sur ce point l'opinion de ses contemporains whigs, il y parvient par des voies bien différentes des leurs et ne partage ni leur engouement pour le modèle républicain classique, ni leur mépris des monarchies absolues, et son analyse du Commonwealth de Cromwell tranche en bien des points avec celle de Harrington. Hume refuse toute systématisation, comme en témoigne le fait que la présente typologie n'est pas établie clairement par lui, et il insiste chaque fois sur l'importance de la prise en compte des traditions et du contexte politique et social. On peut supposer que ce refus participe de sa volonté de ne pas affaiblir l'autorité des régimes en place, souci qui sous-tend l'ensemble de ses écrits politiques. Voilà défini le cadre dans lequel Hume est amené à imaginer son utopie ; il reste à en examiner le contenu.

\section{Le commonwealth idéal selon Hume}

Remarquons tout d'abord que le terme commonwealth est l'un des paradigmes de l'humanisme civique et désigne à la fois le corps politique, l'État, la communauté, la république, la collectivité, sans qu'il existe d'adéquation parfaite avec aucun de ces différents termes ${ }^{16}$. Ce terme ne désigne donc pas nécessairement une république au sens strict mais peut se référer à une monarchie.

22 Hume au début de son essai opère une distinction entre plusieurs modèles d'utopies projectives: il critique la République de Platon et Utopia de Thomas More car, dit-il, "[They] suppose a great reformation in the manners of mankind » et sont donc purement illusoires. En revanche explique-t-il, « The Oceana is the only valuable model of a commonwealth, that has yet been offered to the public " (Essays, p. 514). Il est vrai que Harrington a en commun avec Hume une conception empirique de la science politique: ses généralisations reposent sur un grand nombre d'expériences géographiques et historiques, qu'il s'agisse des cités grecques de la république romaine, de Florence ou de Venise (Harrington écrit dans Oceana: "No man can be a politician unless he be first an historian or a traveller ».) Toutefois Hume porte ailleurs sur Harrington et sur le républicanisme harringtonien un jugement plus négatif, comme dans cette lettre de 1775 à son neveu: "I cannot agree with Mr Millar, that the Republican Form of [Government] is by far the best ". Et d'ajouter à propos de l'auteur d'Oceana : « Harrington is an Author of Genius ; but chimerical ${ }^{17} »$. Le modèle de Commonwealth imaginé par Hume possède en fait de nombreuses ressemblances avec Oceana, mais en même temps Hume critique l'œuvre de Harrington sur trois points fondamentaux : le système de rotation, la loi agraire et enfin la concentration de pouvoir dans le Sénat. 


\section{Le système institutionnel}

Conformément à la tradition républicaine, Hume donne à son commonwealth une structure fédérale qui implique l'existence d'un équilibre entre pouvoirs et promeut la participation des citoyens. Le pays est divisé en cent comtés, chaque comté en cent paroisses, et le système est bicaméral avec des assemblées de comtés et le Sénat. Les membres des paroisses et des comtés élisent chaque année des représentants qui élisent à leur tour parmi eux des magistrats de comté et un sénateur. Les candidats qui échouent à être élus comme sénateurs mais obtiennent un tiers des voix forment l'opposition officielle au sein de la "Cour des compétiteurs ». Les assemblées de comtés sont censées détenir le pouvoir législatif mais toute nouvelle loi doit d'abord être débattue au Sénat. Le Sénat possède également le pouvoir exécutif et choisit par un mode de scrutin complexe inspiré de celui de Venise ou de Malte un Protecteur, deux secrétaires d'État, des commissaires au trésor, et des conseillers d'État chargés des affaires étrangères, de la religion, du savoir, du commerce, des lois, de la guerre et de l'amirauté. En cas d'urgence, ces corps exécutifs peuvent exercer des pouvoirs dictatoriaux pendant six mois. Le pouvoir judiciaire est dévolu aux comtés et au Sénat. Enfin, Hume précise que ce commonwealth sera défendu par une milice calquée sur le modèle suisse et qu'il existera une religion établie, l'Église nationale d'obédience presbytérienne soumise aux autorités civiles : «Without the dependence of the clergy, on the civil magistrates, and without a militia, it is vain to think that any free government will ever have security or stability " (Essays, p. 525).

${ }_{24}$ S'il s'appuie sur la tradition républicaine, Hume s'écarte des modèles antérieurs et ses propositions font écho aux analyses que l'on trouve dans ses autres essais politiques. Il entend éviter le danger de dictature et cherche d'abord à réduire le pouvoir du Sénat: « It appears [...] that in the Oceana, the whole legislature may be said to rest in the senate; which Harrington would own to be an inconvenient form of government... ${ }^{18} »$. C'est pourquoi le Sénat dépend du peuple qui l'élit chaque année, et il est tenu en respect par la Cour des Compétiteurs qui contrôle les finances publiques. Hume veut aussi prévenir l'anarchie et se montre soucieux que le peuple participe à la vie du commonwealth, sans que cela n'engendre de confusion; aussi suggère-t-il de le neutraliser en l'empêchant de s'unir dans de grandes assemblées tumultueuses :

If the people debate, all is confusion : if they do not debate, they can only resolve : and then the senate carves for them. Divide the people into many separate bodies; and then they may debate with safety, and every inconvenience seems to be prevented (Essays, p. 523) ${ }^{19}$.

25 Hume s'efforce donc dans sa République idéale de réduire la représentation du peuple en élevant le cens électoral dans chaque édition successive de son essai : dans la première édition, les électeurs sont tous les détenteurs de francsfiefs et tous ceux qui paient la taxe municipale; dans les éditions suivantes jusqu'en 1768, seuls les détenteurs de francs-fiefs d'une valeur de deux cents livres peuvent voter (il est probable que les troubles wilkites expliquent cette augmentation du cens); dans les dernières éditions, la terre doit rapporter vingt livres de rente par an et les citadins doivent acquitter des taxes à hauteur de cinq cents livres par an. Ainsi qu'il l'écrit dans son essai, l'un des remèdes

Études écossaises, 11 | 2008 
contre l'autorité excessive du Sénat « [is] the great dependence of the senators on the people by annual elections ; and that not by an undistinguishing rabble, like the English electors, but by men of fortune and education » (Essays, p. 524).

${ }_{26}$ D'un point de vue institutionnel enfin, Hume cherche à écarter dans cet essai tout risque de dégénérescence ou de corruption du système constitutionnel, et son texte se rattache en cela à la tradition machiaveloharringtonienne. L'un des dangers principaux à ses yeux est celui que font courir les sectes et les factions - qu'il s'agisse de sectes religieuses ou de partis politiques - suspectés d'être les germes destructeurs de l'État ${ }^{20}$. Le nombre réduit de Sénateurs, l'existence d'une opposition officielle et le pouvoir qu'ont les sénateurs de chasser tout élément factieux répondent à cette inquiétude. De même, le danger de voir éclore des sectes religieuses est réduit par l'établissement d'une église établie de structure démocratique. Toutefois il est important de constater que bien qu'il soit hostile aux partis, Hume, à la différence de Bolingbroke, ne juge ni possible ni même souhaitable de les éliminer complètement: "To abolish all distinctions of party may not be practicable, perhaps not desirable, in a free governement ${ }^{21} »$. Il reconnaît que les divisions partisanes sont dans une large mesure ancrées dans la nature humaine ${ }^{22}$. Les factions ne disparaissent même pas sous un gouvernement despotique: elles deviennent souterraines, ce qui les rend encore plus dangereuses (Essays, p. 60). Hume propose donc dans sa constitution idéale, conformément à ce qu'il écrit dans ses essais consacrés aux partis, de modérer l'ardeur des factions et d'utiliser leur force en les institutionnalisant dans la Cour des Compétiteurs.

27 Enfin, loin de penser, comme les admirateurs des cités antiques, que les républiques doivent être de petite taille, Hume estime qu'une grande nation peut adopter un régime républicain, et que si une grande république est plus difficile à mettre en place, une fois établie, elle offre de meilleures garanties de stabilité qu'une petite :

In a large [republican] government, which is modelled with masterly skill, there is compass and room enough to refine the democracy... At the same time, the parts are so distant and remote, that it is very difficult, either by intrigue, or prejudice, or passion, to hurry them into any measures against the public interest (Essays, p. 528-29).

\section{La société civile}

Si son essai se présente comme la Constitution d'une république idéale, Hume ne s'intéresse toutefois pas qu'aux institutions et qu'à l'État, et c'est en cela aussi qu'il s'écarte, ou plus exactement qu'il donne une nouvelle interprétation du républicanisme classique pour introduire une théorie de la société civile. L'un des principaux reproches que Hume adresse à Harrington est le degré d'égalité morale et matérielle que suppose son utopie, égalité que Hume met en question dans son analyse des passions dans le Traité de la nature humaine. Ainsi que l'explique Christopher Finlay :

Hume's emphasis [...] on pride, humility, love, hatred, esteem and contempt reflects the fact that the social relations with which he was concerned measured differences in the material and cultural possessions belonging to individuals [...] in other words relations of inequality ${ }^{23}$. 
Hume critique explicitement le principe de la rotation car elle oblitère les qualités individuelles : "[Oceana's] rotation is inconvenient, by throwing men, of whatever abilities, by intervals, out of public employments » (Essays, p. 515).

30 En outre, et il s'agit là de l'une de ses principales innovations, Hume conteste la validité et l'universalité de la maxime harringtonienne selon laquelle le pouvoir suit la propriété, maxime qui remporte un très vif succès au XVIII ${ }^{e}$ siècle. Harrington expliquait ainsi la corruption des régimes politiques par des principes économiques, dès que la forme du pouvoir ne correspondait plus à la répartition de la propriété foncière : la balance du pouvoir doit être en faveur d'un seul homme dans une monarchie, d'un petit nombre d'hommes dans une monarchie mixte ou du plus grand nombre dans une république, et la stabilité d'Oceana vient de ce que les propriétaires terriens possèdent les deux tiers environ des terres disponibles ${ }^{24}$. Hume ne rejette pas l'idée d'une corrélation entre propriété et pouvoir politique, mais il la nuance fortement, d'une part en relativisant l'importance de la propriété foncière par rapport à d'autres types de richesses, et de l'autre en infléchissant le caractère déterministe de cette maxime ${ }^{25}$. Il montre ainsi dans l'Histoire d'Angleterre que l'économique n'affecte le politique que si les mœurs et l'opinion dominante à une époque donnée le permettent : citant l'exemple de la loi sur l'aliénation des terres promulguée par Henry VII, il indique qu'elle ne contribua que de manière indirecte à l'affaiblissement du pouvoir des barons et au renforcement $\mathrm{du}$ pouvoir des Communes. Le facteur déterminant fut le changement des mentalités : les grandes découvertes de la fin $\mathrm{du} \mathrm{XV}^{\mathrm{e}}$ siècle excitèrent l'esprit d'entreprise et accrurent les richesses; les membres de la gentry et les marchands se trouvèrent en situation de pouvoir racheter une partie de la propriété foncière de la noblesse, et développèrent l'activité économique et commerciale: ils contribuèrent en fait à l'apparition d'un nouveau type de richesse qui transforma les mentalités et, à terme, l'équilibre politique ${ }^{26}$. En outre, Hume montre que les faits eux-mêmes apportèrent un démenti à la thèse de Harrington: dans Oceana, paru en 1656 sous le Commonwealth, Harrington expliquait que les transferts de propriété en faveur des Communes avaient entraîné un transfert du pouvoir et que le rétablissement de la monarchie était désormais impossible; or en 1660 la dynastie des Stuarts était rétablie sur le trône ${ }^{27}$. Pour Hume, il n'existe pas entre la propriété et le pouvoir une connexion nécessaire mais contingente, qui est médiatisée par l'opinion, notamment par ce qu'il nomme l'«opinion relative au droit de propriété ». Comme il l'écrit dans "Of the First Principles of Government » :

It is sufficiently understood, that the opinion of right to property is of moment in all matters of government. A noted author [Harrington] has made property the foundation of all government; and most of our political writers seem inclined to follow him on that particular. This is carrying the matter too far. [...] It is [...] on opinion only that government is founded (Essays, p. 33-34;32).

31 La troisième grande différence avec le républicanisme harringtonien, d'un point de vue social, est que cette république idéale n'est pas dotée d'une économie agraire mais favorise, par sa taille, le développement du commerce. Elle combine ainsi la vertu républicaine et la prospérité d'une nation commerçante moderne, deux préoccupations très chères aux penseurs éclairés 
écossais. Même si ce souci n'apparaît pas de façon explicite dans «Idea of a Perfect Commonwealth", il est frappant de constater que ce commonwealth suppose des conditions économiques et sociales assez avancées, qu'il garantit les libertés individuelles qui sont nécessaires au développement économique et à l'indépendance politique; et il ne faut pas oublier que cet essai parut avec une série de Political Discourses entièrement voués au développement du commerce. Il est également significatif que contrairement à Harrington, les électeurs chez Hume ne sont pas seulement de petits propriétaires fonciers mais aussi les acteurs de la société commerciale. Comme l'explique John Robertson: «The 'Idea of a Perfect Commonwealth' [...] is compatible, as no actual government has been hitherto, with the 'natural' demands of economic development " (Robertson, 1983, p. 173).

32 L'attitude de Hume à l'égard du régime républicain est en fait très symptomatique de sa philosophie générale et de son impartialité. Alors que le modèle républicain est l'une des principales composantes du discours " néoharringtonien " (celui de l'idéologie du Country Party, des True Whigs et des tories) et sert d'arme aux opposants à l'oligarchie whig régnante, Hume s'efforce de le neutraliser en le dépouillant des qualités qui en font un idéal pour les ennemis de la civilisation commerçante. Il reconnaît ainsi que la république vertueuse est en théorie la forme idéale de gouvernement, et qu'à l'origine, c'est sous un régime républicain que le commerce et la culture ont commencé à fleurir. Mais il montre que cela était dû à l'existence de lois générales, grâce auxquelles la prospérité et la politesse purent se développer, et non pas à l'existence d'une économie agraire, facteur au contraire de mœurs barbares. Il remet ainsi en question l'idée que le développement de la civilisation nécessite que l'on prenne les républiques antiques pour modèles.

\section{Une utopie réalisable?}

33 Ce qui caractérise cette utopie, c'est son caractère réalisable, car, explique Hume, le public ne souhaite pas lire de longues spéculations «vaines et chimériques». C'est le sens du reproche qu'il adresse aux utopies de Platon ou de More - elles sont purement imaginaires car elles supposent une réforme profonde des mœurs humaines - mais aussi à celle de Harrington : sa loi agraire est inapplicable (Essays, p. 514-515). Toutefois, Hume prend de nombreuses précautions pour dissuader tout révolutionnaire de vouloir appliquer ce schéma dans un pays dont les traditions politiques et historiques seraient contraires aux principes exposés. Il est clair que cette utopie est le résultat de l'idéalisation des forces civilisatrices dont Hume a analysé le fonctionnement dans son Histoire et dans ses Essais. Il la conçoit comme un horizon dont les magistrats et les législateurs vont pouvoir chercher à se rapprocher, ce qui va leur permettre d'améliorer les pratiques existantes.

\section{En Grande-Bretagne}

Il est significatif que Hume situe son utopie non pas dans une contrée lointaine, mais en Grande-Bretagne. Son utopie se présente comme une entreprise de rectification des défauts de l'utopie harringtonienne (elle-même située dans une Grande-Bretagne 
idéalisée), et des défauts de la Constitution britannique - que Hume, contrairement aux whigs vulgaires, refuse de juger " parfaite » - en prenant appui sur la Constitution des Provinces-Unies: "That the foregoing plan of government is practicable, no one can doubt, who considers the resemblance that it bears to the commonwealth of the United Provinces, a wise and renowned government » (Essays, p. 526. C'est moi qui souligne).

35 En ce qui concerne le mode de scrutin proposé par Hume, on peut observer que son projet marque une indéniable amélioration par rapport à la situation de la Grande-Bretagne, et notamment à celle de l'Écosse. Et la précision de corrections qu'il apporte montre bien qu'il se soucie des conséquences pratiques de son utopie. Ses recommandations auraient supprimé l'inflation du cens, uniformisé les critères de vote d'un bourg à l'autre (en Angleterre, l'octroi du droit de vote était surtout le résultat de manœuvres électorales et de calculs politiques, la taille de l'électorat variant beaucoup d'un lieu à l'autre), et garanti une véritable représentation en Écosse, alors contrôlée par les grands propriétaires et les conseillers de bourg (en 1788 l'Écosse ne comptait qu'environ 4000 électeurs pour les bourgs et les comtés). Il faudra attendre la réforme électorale de 1832 pour que l'uniformisation que préconise Hume ici soit appliquée à toute la Grande-Bretagne, mais avec un cens fixé à un niveau moins élevé : dans les comtés, le droit de vote sera accordé aux détenteurs de propriétés d'une valeur de dix livres annuelles. Le nombre d'électeurs écossais s'accroîtra ainsi de 1400 \% (Stewart, 1992, p. 285n).

36 En outre, Hume propose dans cet essai un remède pour éviter que la dégénérescence de l'un des trois éléments de la constitution mixte britannique - l'élément aristocratique - ne laisse place au seul affrontement entre le peuple et la monarchie, ce qui ferait à terme courir un danger mortel à la constitution. Hume appelle donc ici comme dans d'autres essais à la restauration d'une puissance intermédiaire aristocratique, et il explique dans son essai quelles sont les étapes concrètes qui permettraient à la Constitution britannique de se rapprocher du modèle idéal : rétablissement du Parlement de Cromwell en rendant la représentation égale et en introduisant un suffrage censitaire dans la Chambre des Communes, retrait des évêques et des Pairs d'Écosse de la Chambre des Lords, augmentation du nombre de Pairs et abolition de la pairie héréditaire au profit de la pairie à vie. Comme le remarque Hume: «By this means the House of Lords would consist entirely of the men of chief credit, abilities, and interest in the nation [...]. Such an aristocracy would be an excellent barrier both to the monarchy and against it $^{28}$ ». Remarquons que l'aristocratie à laquelle Hume songe ici n'est pas la noblesse traditionnelle qu'il critique par ailleurs, mais bien plutôt les représentants des nouvelles couches sociales, le middlingrank ${ }^{29}$.

\section{En Amérique}

«Idea of a Perfect Commonwealth » est donc une utopie qui présente des propositions de réforme concrètes pour la Grande-Bretagne, mais la Grande-Bretagne n'est pas son seul champ d'application. L'influence des Lumières écossaises dans les colonies nordaméricaines est bien connue. Nombre d'universités américaines (Columbia, Philadelphie, Princeton) furent fondées par des émigrés éduqués dans les grandes 
universités écossaises, et nombre de signataires de la Déclaration d'indépendance et de rédacteurs de la Constitution américaine bénéficièrent d'une « éducation écossaise » et avaient donc lu l'Histoire d'Angleterre ou les Essais de Hume : pas moins de 19 signataires de la déclaration finale du Congrès continental de 1776, et des personnalités aussi éminentes que James Madison, Alexander Hamilton, Thomas Jefferson ou James Wilson $^{30}$. Sans conférer à Hume des dons de prescience, il n'est pas absurde de penser qu'en 1752, il voyait les colonies du Nouveau Monde comme l'endroit rêvé pour mettre en œuvre cette réforme des pratiques politiques britanniques ${ }^{31}$. Comme il l'écrit au début de son essai : "Who knows... in some future age, an opportunity might be afforded of reducing the theory to practice, either by dissolution of some old government, or by the combination of men to form a new one, in some distant part of the world?" (Essays, p. 513). Dans une étude restée célèbre, Douglass Adair a montré combien Hume, et notamment son essai «Idea of a Perfect Commonwealth" avait inspiré Madison, en particulier dans le fameux $\mathrm{n}^{\circ} 10$ des Federalist Papers ${ }^{32}$. Madison et les autres révolutionnaires américains prirent sans doute conscience en lisant Hume que la virtù républicaine n'était pas nécessairement synonyme d'austérité mais qu'elle était compatible avec le développement du commerce pour peu que des institutions solides fussent établies, que la capacité de nuisance des factions pouvait être atténuée, et surtout que la thèse très en vigueur à l'époque, et défendue notamment par Montesquieu, selon laquelle seule une république de petite taille pouvait être vertueuse, était erronée : "Though it is more difficult to form a republican government in an extensive country than in a city ; there is more facility, when once it is formed, of preserving it steady and uniform, without tumult or faction » (Essays, p. 527).

38 Cependant il n'est pas impossible qu'Adair ait quelque peu exagéré l'influence directe de Hume sur Madison. Il est vrai que Hume était considéré avec méfiance en Amérique en raison de son scepticisme religieux, de son refus de condamner les Stuarts dans son Histoire d'Angleterre - refus qui lui valait la réputation de tory - et de sa défense du luxe et de la richesse, sources de corruption aux yeux des républicains classiques. Thomas Jefferson accusera d'ailleurs Hume de lui avoir "pollué » l'esprit et s'élèvera contre l'hostilité humienne aux principes démocratiques :

I fear nothing for our liberty from the assaults of force; but I have seen and felt much and fear more from English books... When I look around me for security against these seductions, I find it in the widespread of our agricultural citizens, in their unsophisticated minds... and their power, if called on, to crush the Humists of our cities, and to maintain the principles which severed us from England ${ }^{33}$.

39 Il est possible que d'autres auteurs écossais, notamment Hutcheson, que John Witherspoon fit découvrir à Madison à l'université de Princeton, aient joué un rôle au moins aussi important que Hume, lui-même influencé par Hutcheson $^{34}$. Quoi qu'il en soit, en appliquant le républicanisme modéré de Hume au contexte américain, et en tenant compte de l'expérience respective de la Grande- Bretagne et de ses colonies, Madison et les autres pères de la Constitution américaine se révèlent fidèles aux leçons de Hume. Il est significatif que les FederalistPapers s'achèvent d'ailleurs sur la citation suivante de Hume, qualifié de « equally solid and ingenious writer» :

To balance a large state or society, whether monarchical or republican, on general laws, is a work of so great difficulty, that no human genius, however comprehensive, is able, by the mere dint of reason and reflection, to effect it. The 
judgments of many must unite in this work; Experience must guide their labour :

Time must bring it to perfection... ${ }^{35}$

\section{Le réformisme de Hume}

Hume nous propose donc une utopie qui, tout en s'inscrivant dans la tradition républicaine, fait également évoluer cette tradition en tenant compte des réalités de son temps. La préférence de Hume pour la république peut sembler paradoxale si l'on songe que c'est un régime étroitement associé à l'idéologie passéiste des True Whigs et au discours néoharringtonien qu'il critique par ailleurs. Mais contrairement aux républiques antiques ou à l'utopie harringtonienne, la république idéale de Hume est une grande république commerciale. Si Hume choisit un modèle républicain, c'est parce qu'il constate que les progrès accomplis par la civilisation européenne sous l'effet du développement du commerce vont dans un sens républicain, en accroissant la circulation des biens et des idées, et en renforçant la liberté et la culture. Or, cette montée en puissance des éléments républicains dans des régimes déjà constitués n'est pas exempte de problèmes, surtout dans le cadre d'une constitution mixte comme la constitution britannique. Loin d'être une satire, une pure utopie, ou un simple jeu d'esprit, la république humienne se fonde sur l'observation des principaux problèmes constitutionnels rencontrés par les régimes européens et leur apporte une solution: elle s'efforce de concilier le principe républicain de liberté et le principe monarchique d'autorité dans le cadre d'un grand État-nation, et d'échapper à l'instabilité chronique qui caractérise la constitution britannique.

${ }_{41}$ Ce que nous révèle cette utopie sur la science politique de Hume, c'est qu'il s'efforce d'améliorer ce qui est, en tenant compte de ce qui a été fait et de ce qu'il est humainement possible de faire. La politique humienne peut être décrite comme réaliste, non parce qu'elle est entièrement soumise à la réalité réalité dont Hume montre bien dans sa théorie de la connaissance qu'elle n'est pas un donné, mais qu'elle est en partie construite - mais parce que cette politique reconnaît la nécessité d'un mouvement dialectique entre la «vie commune » et la réflexion critique.

42 Un autre aspect du réalisme utopique de Hume tient à la redéfinition du rapport entre l'idéal et le réel qu'il propose. L'utopie se situe dans un lieu idéal, mais elle n'est pas purement imaginaire. Idéalisation du réel, elle peut constituer un modèle parfait, inaccessible, qui permet une fuite hors de la réalité ; ou bien elle peut se présenter comme un ordre nouveau que l'on va vouloir instituer au prix d'un renversement complet de l'ordre existant: l'utopie devient alors une force révolutionnaire susceptible de détruire la réalité qui l'a produite ${ }^{36}$.

43 L'utopie humienne est réaliste dans la mesure où Hume réfute aussi bien l'existence d'un modèle parfait d'organisation politique, que l'idée que l'on veuille risquer de bouleverser l'ordre existant pour se conformer à des valeurs transcendantes imaginaires. Contrairement à d'autres utopistes, Hume a un rapport dialectique au réel, et il cherche avant tout à explorer et comprendre le réel dans toute sa complexité, sans s'en détourner. D'où sa méfiance à l'égard des théories politiques qui s'appuient sur des maximes à valeur universelle et absolue. Hume insiste plutôt sur la variété et sur la relativité de telles maximes, 
sa science politique s'appuyant sur une étude empirique de la nature humaine et sur l'observation des phénomènes historiques. Son rejet de tels modèles de pensée le conduit à formuler une politique de la modération qui exclut toute forme d'extrémisme.

44 Pour autant, le scepticisme et l'empirisme humiens ne signifient pas que Hume juge absurde ou inutile de s'interroger sur la façon d'améliorer le réel. Par le choix du titre "Idea of a Perfect Commonwealth» (plutôt que «The Perfect Commonwealth »), Hume montre qu'il s'intéresse avant tout au concept de république idéale et à ses rapports avec la réalité, et que cette république idéale n'est pas un absolu. La fin de l'essai souligne bien le caractère relatif de son régime idéal :

It is a sufficient incitement to human endeavours, that such a government would flourish for many ages; without pretending to bestow, on any work of man, that immortality, which the Almighty seems to have refused to his own productions.

(Essays, p. 529)

45 Cette « utopie » entretient donc des rapports très étroits avec la réalité et Hume espère orienter l'action des législateurs et susciter un débat sans pour autant exciter des passions révolutionnaires. C'est sans doute ce qui explique que cette utopie ait trouvé en Amérique des applications concrètes qui allaient sans doute au-delà de ce que pouvait espérer le philosophe écossais. La modestie de l'approche de Hume ne l'empêche donc pas d'être visionnaire. L'enjeu de la politique de Hume est de ne voir dans l'état présent des choses ni le fruit d'une évolution inéluctable, ni le résultat d'une volonté supérieure, mais un donné produit par une interaction complexe de facteurs, ce qui interdit toute velléité de retour en arrière, et toute projection trop rapide dans l'avenir. L'absence de norme absolue n'est pas un obstacle à la réforme politique, bien au contraire : elle rend la réforme concevable.

\section{BIBLIOGRAPHIE}

ADAIR Douglass, “'That Politics May be Reduced to a Science' David Hume, James Madison, and the Tenth Federalist », Huntington Library Quarterly n 20, 1956-1957, p. 343-360.

Anon., Memoirs concerning the life and manners of Captain Mackheath. London, 1728, $61 \mathrm{p}$.

ARKIN Marc M., «'The Intractable Principle' David Hume, James Madison, Religion and the Tenth Federalist », American Journal of Legal History n³9, 1995, p. 149-176.

BOCCARA Nadia, « Anti-Utopian Realism in David Hume », Studies on Voltaire and the Eighteenth Century n 303, 1992, p. 404-408.

BOLINGBRoKE Henry St John, Viscount of. The Idea of a Patriot King, 1740, vol. 2 of The Works of Lord Bolingbroke, 4 vols, 1844, London : F. Cass, 1967. 
BURGH James, An Account of the First Settlement, Laws, Form of Government, and Police, of the Cessares, A People of South America, London, 1764.

CLAEYS Gregory, ed. \& intro., Utopias of the British Enlightenment, Cambridge, Cambridge UP, 1994. CONNIFF James, « The Enlightenment and American Political thought : A Study of the origins of Madison's Federalist Number 10 », Political Theory, vol. 8, n³ , Aug. 1980, p. 381-402.

-, « Hume's Political Methodology : A Reconsideration of ‘That Politics May Be Reduced to a Science' ", Review of Politics, vol. 38, n 1, 1976, p. 88-108.

DEFOE Daniel, The Consolidator : or Memoirs of Sundry Transactions from the World in the Moon. London, 1705.

FINLAY Christopher J., " Hume's Theory of Civil Society », European Journal of Political Theory, vol. 3, $\mathrm{n}^{\circ}$ 4, 2004, p. 369-391.

FORBES Duncan, Hume's Philosophical Politics, Cambridge : Cambridge UP, 1975.

GAUTIER Claude, ed. Hume et le concept de société civile, Paris, PUF, 2001.

GERALD Stourzh, Alexander Hamilton and the Idea of Republican Government (Stanford : Stanford UP, 1970).

HARRINGTON James, The Commonwealth of Oceana and A System of Politics, ed. \& intro. J.G.A. Pocock, Cambridge, Cambridge UP, 1992.

HOLMESBY John, The voyages, travels, and wonderful discoveries of Capt. John Holmesby. Containing a series of the most surprising and uncommon events, which befel the author in his voyage to the Southern Ocean, in the year 1739, London, 1757.

HONT Istvan \& MICHAEL Ignatieff, eds., Wealth and Virtue : the Shaping of Political Economy in the Scottish Enlightenment, Cambridge : CUP, 1983.

HUME David, Essays Moral Political and Literary, 1741-1777, ed. E.F. Miller, Indianapolis, LibertyClassics, 1985.

-, Essais, moraux, politiques et littéraires et autres essais, 1741-1777, ed. \& trad. Gilles Robel, Paris, PUF, 2001.

-, The History of England, from the Invasion of Julius Caesar to the Revolution in 1688, 1754-1778, Pref. W.B. Todd, 6 vols, Indianapolis, LibertyClassics, 1983

LANGFORD Paul, A Polite and Commercial People : England 1727-1783, Oxford, OUP, 1992, 803 p.

LIVINGSTON D.W., « Hume and America », The Kentucky Review, vol. 4, n 3, spring 1983, p. 15-37.

LOCKE John, « Second Treatise of Government », Two Treatises of Government, ed. P. Laslett, 1690, Cambridge, Cambridge UP, 1988.

MOORE James, « Hume's Political Science and the Classical Republican tradition ». Canadian Journal of Political Science, vol. 10, $\mathrm{n}^{\circ}$ 4, Dec. 1977, p. 809-839.

MORGAN Edmund S., « Safety in Numbers : Madison, Hume and the Tenth Federalist », Huntington Library Quarterly 49, 1986, p. 95-110.

PINCUS Steve, « Neither Machiavellian Moment nor Possessive Individualism : Commercial Society and the Defenders of the English Commonwealth », American Historical Review, vol. 103, n 3, 1998, p. 705-736. 
Pососк J.G.A., « Civic Humanism and its Role in Anglo-American Thought », Politics, Language and Time, New York, Atheneum, 1973, p. 80-103.

ROBEL Gilles, Lumières et conservatisme dans la pensée politique de David Hume, Lille : P.U. du Septentrion, 1999.

Rотн Robert J., « The Empiricism of Hume's Political theory », International Philosophical Quarterly, vol. XXXI.4, n 124, Dec. 1991, p. 403-417.

scotT Jonathan, « What Were Commonwealth Principles?», Historical Journal, vol. 47, $\mathrm{n}^{\circ}$ 3, 2004, p. 591-613.

STEWART John B., Opinion and Reform in Hume's Political Philosophy, Princeton, Princeton UP, 1992.

STOURZH Gerald, Alexander Hamilton and the Idea of Republican Government, Stanford, Stanford UP, 1970.

\section{NOTES}

1. Daniel Defoe, The Consolidator: or Memoirs of Sundry Transactions from the World in the Moon (London, 1705) ; Memoirs Concerning the Life and Manners of Captain Mackheath(London, 1728) p. 10 ; John Holmesby, The Voyages, Travels, and Wonderful Discoveries of Capt. John Holmesby. Containing a series of the most surprising and uncommon events, which befel the author in his voyage to the Southern Ocean, in the year 1739 (London, 1757) notamment les chapitres 13 et 14 sur l'Angleterre, p. $142-176$.

2. Voir Istvan Hont \& Michael Ignatieff, Wealth and Virtue: the Shaping of Political Economy in the Scottish Enlightenment (Cambridge : CUP, 1983).

3. C'est par exemple le cas dans An Account of the First Settlement, Laws, Form of Government, and Police, of the Cessares, A People of South America du calviniste républicain écossais James Burgh (1764).

4. «Milton's political economy, and indeed that of Harrington, appears quite conservative ». Steven Pincus, 1998, p. 707.

5. J. G. A. Pocok, «Introduction », The Commonwealth of Oceana, 1992, p. 17.

6. Concernant la position de Hume sur l'échiquier politique de son temps, voir Gilles Robel, 1999, p. 660-677.

7. Alexander Pope, Essay on Man (London, 1732-34) Bk 3.

8. David Hume, « Of the Rise and Progress of the Arts and Sciences », Essays 117.

9. David Hume, History ch. LXI, VI : 74.

10. Sur le "whiggisme vulgaire» ou whiggisme doctrinaire, voir Duncan Forbes, Hume's Philosophical Politics, Cambridge : CUP, 1975, p. 139 sq.

11. John Locke, "Second Treatise of Government", 1690, §90-91, p.326. Voir également Bolingbroke, The Idea of a Patriot King, 1740, vol. II, p. 387.

12. David Hume, "Of the Rise and Progress of the Arts and Sciences », Essays, p. 125.

13. Il s'agit là d'une remise en cause de l'idéologie dominante anglaise, comme Hume l'explique dans l'une de ses lettres à l'abbé Le Blanc ("You wou'd have remark'd in my Writings, that my Principles are, all along, tolerably monarchical, \& that I abhor, that low Practice, so prevalent in England, of speaking with Malignity of France », 12 sept. 1754), Letters vol. I, p. 194.

14. David Hume, « Of the Parties of Great Britain », Essays 614.

15. David Hume, History ch. LX, vol. VI, p. 39-41.

16. Locke explique ainsi qu'il entend désigner par le terme Common-wealth: "non une démocratie, ni aucune forme de gouvernement, mais toute communauté indépendante que les Latins 
appellent du nom de civitas; le terme qui y correspond le mieux dans notre langue, c'est commonwealth : il exprime très exactement une telle société d'hommes, ce que ne font pas les mots de community ou de city en anglais, car il peut exister des communautés subordonnées dans un gouvernement; et chez nous, le mot city possède un sens très différent de celui de commonwealth. » John Locke, Second traité du gouvernement, trad. J.-F. Spitz (Paris : PUF, 1994) chap. X $§ 133$ p. 95.

17. David Hume, « Letter to David Young the Younger, 8 déc. 1775 », Letters vol. 2, p. 306.

18. David Hume, «Idea of a Perfect Commonwealth », Essays 516. Dans la lettre citée ci-dessus, Hume explique à son neveu : "As the People have only a negative, the Senate woud perpetually gain Ground upon them ». Letters vol. II, p. 307.

19. Dans l'essai «That Politics May Be Reduced to a Science » (1741) Hume expliquait déjà que le pouvoir législatif devait consister en un corps représentatif plutôt qu'en une assemblée populaire (Essays p. 16-17). Dans "Of the First Principles of Government» (1741), il explique que les représentants doivent tenir compte des désirs de leurs administrés, mais garder une marge d'indépendance et éviter d'être de simples mandataires (Essays, p.35-36). Sur le fanatisme populaire sous Cromwell, voir David Hume, History vol. V, p. 545-546.

20. Voir l'essai «Of Parties in General » (1741) : «Factions subvert government, render laws impotent, and beget the fiercest animosities among men of the same nation, who ought to give mutual assistance and protection to each other ». Essays p. 55. Notons que Hume ne met pas tous les partis sur le même plan: il distingue les factions de personne (personal factions) des partis fondés sur des questions « réelles" (real factions). Ces derniers se subdivisent en trois catégories : les partis d'intérêt, les partis de principe et enfin les partis d'affection. Ce sont les partis de principe qui sont à ses yeux les plus dangereux.

21. David Hume, « Of the Coalition of Parties ", Essays 493.

22. "Such is the nature of the human mind, that it always lays hold on every mind that approaches it ; and as it is wonderfully fortified by an unanimity of sentiments, so is it shocked and disturbed by any contrariety » David Hume, « Of Parties in General », Essays p. 60-61.

23. Christopher J. Finlay, 2004, p. 372. Voir également Claude Gautier, 2001.

24. James Harrington, Oceana $100 \mathrm{sq}$.

25. Il faut noter cependant qu'à l'époque où Harrington formule sa théorie - en pleine guerre civile - ce déterminisme est une forme de détachement scientifique, au moment où les factions rivales se déchirent sur la question de savoir quel principe métaphysique confère à un gouvernement sa vraie légitimité. Didier Deleule, 1979, p. 338.

26. David Hume, History, ch. XXVI, III : 80. Voir aussi James Moore, 1977, p. 816.

27. " HARRINGTON thought himself so sure of his general principle, that the balance of power depends on that of property, that he ventured to pronounce it impossible ever to re-establish monarchy in England: But his book was scarcely published when the king was restored ». David Hume, "Whether the British Government inclines more to Absolute Monarchy or to a Republic ", Essays 47.

28. Essays, p. 527. Il est difficile de ne pas trouver à l'abolition de la pairie héréditaire une résonance très actuelle!

29. Sur la critique humienne de la noblesse traditionnelle, voir notamment « Of the Middle Station of Life ", Essays, p. 545-552 ou History, vol. III, p. 76-77 et Gilles Robel, 1999, p. 555sq.

30. Gerald Stourzh, Alexander Hamilton and the Idea of Republican Government (Stanford: Stanford UP, 1970) ch. 2 \& 3 ; Donald Livingston, 1983.

31. David Hume, Essays p.513. Il écrit ainsi dans le volume de l'History consacré aux Stuarts (rédigé au début des années 1750) : «What chiefly renders the reign of James memorable, is the commencement of the English colonies in America; colonies established on the noblest footing that has been known in any age or nation... The spirit of independency, which was reviving in England, here shone forth in its full lustre, and received new accession from the aspiring character of 
those, who, being discontented with the established church and monarchy, had sought for freedom amidst those savage deserts. » David Hume, History, V : 146-47. C'est moi qui souligne.

32. Douglass Adair, 1956-1957, p. 349sq. Adair a montré que Madison a même emprunté certains passages tirés d'essais de Hume.

33. Thomas Jefferson, "Letter to H. G. Spafford ", 17 March 1814, cité dans Thomas Jefferson on Democracy, ed. Saul K. Padover (New York : Mentor Books, 1946) 85.

34. James Conniff, 1980. Voir aussi Edmund S. Morgan, 1986 et Marc M. Arkin, 1995.

35. David Hume, "Of the Rise and Progress of Arts and Sciences ", Essays 124 cité explicitement à la fin du Federalist Paper $\mathrm{N}^{\circ} 85$.

36. Nadia Boccara, 1992, p. 405.

\section{AUTEUR}

\section{GILLES ROBEL}

Université Paris-Est Marne-la-Vallée 